\title{
ESTOQUE DE CARBONO DO SOLO EM SISTEMAS VEGETAIS COM MANEJO AGRÍCOLA DIFERENCIADO NO OESTE PAULISTA
}

\author{
Isabela Marega Rigolin ${ }^{1}$, Carlos Henrique dos Santos ${ }^{2}$, Juliano Carlos Calonego ${ }^{2}$, Carlos Sergio \\ Tiritan $^{2}$

\footnotetext{
${ }^{1}$ Mestranda do Programa de Pós-Graduação em Agronomia, Docente da Faculdade de Engenharia da UNOESTEPresidente Prudente-SP. ${ }^{2}$ Docente, Programa de Pós-Graduação em Agronomia, UNOESTE-Presidente Prudente-SP. Correspondência para: Isabela Marega Rigolin - isabelarigolin@unoeste.br
}

\section{RESUMO}

Este trabalho teve como objetivo verificar o teor de matéria orgânica de um Argissolo Vermelho distroférrico sob diferentes sistemas de manejo de culturas agrícolas, bem como o reflexo direto destes sistemas sobre o teor de matéria orgânica, o estoque de carbono orgânico e a densidade do solo. O experimento foi conduzido na Universidade do Oeste Paulista (UNOESTE), em Presidente Prudente-SP. Os sistemas de manejo foram selecionados de acordo com o histórico de uso das áreas: $\underline{\mathrm{MN}}$ - área de mata nativa; $\underline{\mathrm{EUC}}$ - área de eucalipto; $\underline{\mathrm{SSD}}$ - área manejada no sistema de semeadura direta; PSTI - área de pastagem de Panicum maximum cv. Mombaça manejada com irrigação; PST - área de pastagem de Urochloa brizantha sem manejo específico. O delineamento experimental foi caracterizado como inteiramente casualizado, estruturado em parcelas subdivididas, onde as parcelas foram constituídas pelos sistemas de manejo e as subparcelas foram representadas pelas profundidades $(0-5,5-10,10-20$ e 20-40 cm) de solo amostrado nas entrelinhas de plantio. Após a coleta, as amostras foram transportadas para o laboratório para a determinação do teor de matéria orgânica (MOS) e carbono orgânico (COS), densidade aparente (Ds) e cálculo do estoque de carbono total do solo (ECOT) com base na massa equivalente de solo. Os resultados obtidos foram submetidos à análise de variância e ao teste de comparação de médias ao nível de $5 \%$ de probabilidade. A retirada da vegetação nativa proporcionou aumento da Ds e a redução da MOS e do ECOT do solo. Os diferentes sistemas de manejo de culturas agrícolas não melhoraram os valores dos atributos Ds e MOS. O manejo de culturas em SSD apresentou maior ECOT na profundidade de $20-40 \mathrm{~cm}$.

Palavras-chave: matéria orgânica; densidade do solo; plantio direto.

\section{SOIL CARBON STOCK IN SOIL PLANT SYSTEMS WITH DIFFERENT AGRICULTURAL MANAGEMENT IN THE WEST OF SÃO PAULO STATE}

\begin{abstract}
This study aimed to determine the organic matter content of a dystrophic Ultisol under different tillage systems and crop direct reflection of these systems on the soil organic matter content (OMS), stock of organic carbon (SOC) and bulk density (BD). The experiment was conducted at UNOESTE in Presidente Prudente-SP. The management systems were selected according to the historical use of areas: NF - native forest; EUCA - area of eucalyptus ; NTA - area managed in notillage; PSWI - area of pasture grass Panicum maximum cv. Mombasa managed with irrigation; PST - area of pasture Urochloa brizantha without specific management. The experiment was described as randomized, structured in a split plot, where the plots represented the management systems and subplots represented the sampled depths $(0-5,5-10,10-20$ and $20-40 \mathrm{~cm})$ of soil sampled between the rows of crops. After sampling, the samples were transported to the laboratory soil analysis for determination the content of organic matter (OMS) and organic carbon (COS), bulk
\end{abstract}


density (BD) and calculating the total stock of soil carbon (TOCS) based on equivalent soil mass. The results obtained were subjected to analysis of variance and comparison of means at $5 \%$ probability test. The removal of native vegetation provided an increase in BD and reduction in OMS and soil TOCS. The management of agricultural crops did not improve the attributes BD and SOM. The crop management SSD showed higher TOCS at a depth of 20-40 cm.

Keywords: organic matter; bulk density; no tillage. 


\section{INTRODUÇÃO}

Os sistemas de uso e manejo dos solos podem influenciar a sustentabilidade e a produtividade dos ecossistemas agrícolas, por meio da degradação de suas propriedades químicas, físicas e biológicas; as quais determinam o desenvolvimento das culturas, com impactos na produtividade e no meio ambiente (PAGLIAI; VIGNOZZI; PELLEGRINI, 2004). O solo é um sistema aberto, com permanente troca de matéria e energia com o meio (ADDISCOTT, 1995), o que é considerado complexo, em virtude de uma intrincada rede de relações entre os subsistemas que o compõem, representados pelos vegetais, organismos e matéria mineral (LOVATO et al., 2004).

De fato, a conversão de ecossistemas naturais em sistemas agrícolas associada ao uso intensivo do solo, envolve uma série de atividades que afetam as taxas de adição e decomposição da matéria orgânica do solo - MOS (ZINN; LAL; RESCK, 2005), propiciando condições para o início da sua degradação química e física. Este componente do processo de degradação geralmente está associado ao processo químico de perda de matéria orgânica, além da integridade estrutural dos solos. Como consequência, há alterações nas propriedades do solo que controlam processos fundamentais relacionados à dinâmica de água, ar e calor (FEDDEMA, 1998).

Atributos físicos como a densidade, ligada diretamente à estabilidade estrutural do solo (STONE; SILVEIRA, 2001), e os químicos como o teor de matéria orgânica e o estoque de carbono orgânico total são utilizados, dentre outros, para estudar os impactos do uso e manejo na qualidade química e física do solo (VIANA et al., 2011).O C orgânico do solo não é uma propriedade física, mas influencia direta e indiretamente a maioria delas, de modo que a sua redução está relacionada com a degradação física dos solos (CARDOSO; POTTER; DEDECEK, 1992).

Portanto, o monitoramento das alterações da MOS tem sido um indicador dos efeitos do manejo e uso do solo (CONCEIÇÃO, 2008), principalmente em solos de textura arenosa, como os argissolos, considerados altamente vulneráveis estruturalmente (BLANCO-CANQUI et al., 2009) e suscetíveis à erosão (OLIVEIRA, 1999). A MOS interage com minerais no solo formando complexos organominerais por meio de diversos mecanismos de interação, resultando em partículas secundárias de diversos tamanhos e formas, desde micro até macroagregados de alguns milímetros de tamanho (TISDALL; OADES, 1982).

Além disso, é crescente a preocupação mundial em relação às 
mudanças do clima no planeta, que na maioria das vezes são decorrentes, principalmente, das emissões de $\mathrm{CO}_{2}$ e outros gases de efeito estufa como o metano $\left(\mathrm{CH}_{4}\right)$ e o óxido nitroso $\left(\mathrm{N}_{2} \mathrm{O}\right)$. Neste contexto, no caso do $\mathrm{CO}_{2}$, torna-se imperativo a redução de sua emissão bem como a maximização do seu sequestro da atmosfera. Sistemas agrosilvipastoris manejados adequadamente podem potencializar ou mitigar a emissão de $\mathrm{CO}_{2}$ para a atmosfera, bem como a semeadura direta sobre a palha (SSP), a integração lavoura-pecuária-floresta, a adoção de reflorestamentos, o manejo adequado de pastagens e a colheita mecanizada da cana-de-açúcar, entre outros fatores, que também podem aumentar consideravelmente os estoques de $\mathrm{C}$ no solo provenientes da atmosfera (CARVALHO et al., 2010).

Este trabalho teve como objetivo verificar o teor de matéria orgânica de um solo Argissolo Vermelho distroférrico sob diferentes sistemas de manejo de culturas agrícolas e o reflexo direto destes sistemas sobre o estoque de carbono orgânico e a densidade do solo.

\section{MATERIAL E MÉTODOS}

O experimento foi realizado na Universidade do Oeste Paulista (UNOESTE), em Presidente Prudente, São Paulo, em solo classificado como Argissolo Vermelho distroférrico de textura média (EMBRAPA, 2006).

As áreas com sistemas de manejo específico foram selecionadas de acordo com o histórico de uso e importância regional e estão descritas a seguir: Área 1: $\underline{\text { SSD }}$ - Área de rotação (milho-aveia/milho-Urochloa brizantha/milho) manejadas no sistema de semeadura direta (SSD) à 3 anos agrícolas; Área 2: EUC - Área de Eucalipto (Eucaliptus grandis) com 3 anos de manejo convencional; Área 3: $\underline{\text { PST }}$ - Pastagem de Urochloa brizantha sem manejo específico à 3 anos; Área 4: PSTI - Pastagem de capim Mombaça (Panicum maximum cv. Mombaça) manejada com irrigação à 3 anos; Área 5: $\mathrm{MN}$ - Área de Mata Nativa, sem a intervenção humana à 50 anos.

O delineamento experimental utilizado foi caracterizado como inteiramente casualizado (DIC), em esquema fatorial, estruturado em parcelas subdivididas onde as parcelas foram representadas pelo sistema de manejo vegetal e as subparcelas foram representadas pelas profundidades de solo amostradas (0-5, 5-10, 10-20 e 20-40 cm). As amostras de terra coletadas (deformadas e indeformadas) foram consideradas como repetições, não se caracterizando, portanto, como repetição espacial. 
Nas áreas escolhidas, para a obtenção dos valores de densidade aparente (Ds), foram coletadas amostras indeformadas de solo em trincheira com dimensão de 1,0 m de largura x 1,0 m de profundidade, aberta no centro de cada parcela experimental. Utilizando-se cilindros de aço inox (anéis volumétricos) com volume de $98 \mathrm{~cm}^{3}(5,0 \mathrm{~cm}$ de altura $x 5,0 \mathrm{~cm}$ de diâmetro interno) foram coletadas três amostras indeformadas em cada camada/parcela, totalizando 60 amostras. As amostras foram colocadas para secar em estufa com circulação forçada de ar, a $105{ }^{\circ} \mathrm{C}$, e retiradas após 48 horas para pesagem em balança semi-analítica, com duas casas decimais e precisão de 0,001 g, para a obtenção da massa de solo seco. 0 cálculo da Ds foi realizado utilizando a equação: Densidade aparente $(D s)=\mathrm{m} / \mathrm{v}$, onde $\mathrm{m}=$ massa de solo seco $(\mathrm{g}) ; \mathrm{v}=$ volume do anel de aço inox $\left(\mathrm{cm}^{3}\right)$ (EMBRAPA, 1997).

A determinação de carbono orgânico (COS) foi realizada através da coleta de amostras deformadas de solo, nas entrelinhas dos cultivos e nas profundidades de 0-5, 5-10, 10-20 e 20-40 cm. Foram coletadas cinco subamostras que foram misturadas para compor três amostras compostas em cada parcela e profundidade, sendo considerada cada amostra como repetição. No total, foram coletadas 60 amostras, as quais foram submetidas ao método de análise de Walkley-Black (1934) descrito em Raij et al. (2001). O teor de matéria orgânica do solo (MOS) obtido por este método foi convertido em carbono orgânico total (COS) pela aplicação do fator de Van Bemmelen (1,724), com base no pressuposto de que a matéria orgânica do solo contém $58 \%$ de $\mathrm{C}$ orgânico. Portanto, o valor de MOS foi dividido por 1,724.

O estoque de carbono orgânico total no solo (ECOT) nas amostras deformadas foi calculado em cada área com base na massa equivalente de solo (ELBERT; BETTANY, 1995) de acordo com a seguinte equação: ECOT $\left(\mathrm{Mg} \mathrm{ha}^{-1}\right)=\{$ Teor de $\mathrm{C} \times \mathrm{E} \times \mathrm{D}\}$, em que $\mathrm{C}=$ teor de carbono em $\mathrm{g} \mathrm{kg}^{-1}, \mathrm{E}=$ espessura da camada $(\mathrm{cm})$ e $D=$ densidade aparente do solo $\left(\mathrm{g} \mathrm{cm}^{-3}\right)$ na camada. Posteriormente, seguindo recomendação de SISTI et al. (2004), o ECOT dos sistemas de manejo, exceto a área de Mata Nativa (MN), foram corrigidos para uma mesma massa de solo, tendo as condições de $\mathrm{MN}$ como referência de valores.

Os parâmetros obtidos foram submetidos à análise de variância e as médias calculadas foram comparadas pelo teste de Tukey, ao nível de $5 \%$ de probabilidade. A significância da interação entre os fatores sistemas de manejo vegetal e profundidades de solo também foi verificada pelo teste Tukey, ao nível de $5 \%$ de probabilidade. 


\section{RESULTADOS E DISCUSSÃO}

O sistema de manejo vegetal influenciou os valores de densidade (Ds), matéria orgânica (MOS) e estoque de carbono orgânico total do solo (ECOT) (Tabela 1).

Tabela 1. Valores de F calculados para densidade aparente (Ds), matéria orgânica (MOS) e estoque de carbono total (ECOT) do solo nos cinco sistemas de manejo e nas quatro profundidades avaliadas.

\begin{tabular}{lccc}
\hline \multirow{2}{*}{ Tratamentos } & \multicolumn{3}{c}{ Valores do Teste $\mathrm{F}$} \\
\cline { 2 - 4 } & Ds & MOS & ECOT \\
\hline Sistemas de Manejo (a) & $7,48^{* *}$ & $5,69^{* *}$ & $5,36^{*}$ \\
Profundidade (b) & $0,51^{\text {ns }}$ & $1,30^{\text {ns }}$ & $43,70^{* *}$ \\
Interação (a) $\times(b)$ & $1,02^{\text {ns }}$ & $1,03^{\text {ns }}$ & $2,47^{*}$ \\
\hline
\end{tabular}

** significativo ao nível de $1 \%$ de probabilidade $(p<0,01)$; ${ }^{*}$ significativo ao nível de $5 \%$ de probabilidade $(p<0,05)$; ns não significativo.

Além disso, os resultados apresentados na Tabela 1 indicam que a profundidade de amostragem do solo também apresentou alterações nos valores de ECOT, na mesma avaliação. Houve apenas uma interação significativa, a qual foi constatada entre os sistemas de manejo e a profundidade de coleta das amostras para o parâmetro ECOT.

O menor valor de Ds bem como os maiores teores de MOS e ECOT foram encontrados nas amostras coletadas da área de MN (Tabela 2), enquanto que os sistemas vegetais agrícolas (SSD, EUC, PST e PSTI) apresentaram Ds significativamente superior à $\mathrm{MN}$, além de valores significativamente menores de MOS e ECOT comparado à mesma área. Entre as áreas com manejo agrícola, houve diferença estatística apenas para a Ds. 
Tabela 2. Densidade aparente (Ds), teor de matéria orgânica (MOS) e estoque de carbono orgânico total (ECOT) do solo em cinco sistemas de manejo avaliados.

\begin{tabular}{cccc}
\hline $\begin{array}{c}\text { Sistema de } \\
\text { Manejo }\end{array}$ & $\begin{array}{c}\text { Ds } \\
\left(\mathrm{g} \mathrm{cm}^{-3}\right)\end{array}$ & $\begin{array}{c}\text { MOS } \\
\left(\mathrm{g} \mathrm{dm}^{-3}\right)\end{array}$ & $\begin{array}{c}\text { ECOT } \\
\left(\mathrm{Mg} \mathrm{ha}^{-1}\right)\end{array}$ \\
\hline SSD & $1,49 \mathrm{a}$ & $12,67 \mathrm{~b}$ & $11,47 \mathrm{~b}$ \\
EUC & $1,38 \mathrm{~b}$ & $13,25 \mathrm{~b}$ & $8,90 \mathrm{~b}$ \\
PST & $1,31 \mathrm{~b}$ & $15,33 \mathrm{~b}$ & $8,52 \mathrm{~b}$ \\
PSTI & $1,32 \mathrm{~b}$ & $15,21 \mathrm{~b}$ & $9,26 \mathrm{~b}$ \\
MN & $1,19 \mathrm{c}$ & $31,37 \mathrm{a}$ & $21,32 \mathrm{a}$ \\
\hline CV (\%) & 9,24 & 77,40 & 37,79 \\
DMS & 0,16 & 12,86 & 10,85 \\
\hline
\end{tabular}

Letras iguais, minúsculas na coluna não diferem entre si a $5 \%$ de probabilidade pelo teste Tukey.

Os manejos das culturas avaliados nas áreas agrícolas não permitiram mudança significativa nos atributos químicos do solo apresentados na Tabela 2. Ao se comparar os valores de MOS e ECOT observa-se que não existiu diferença estatística entre os manejos e que os mesmos foram inferiores comparados à área de MN. Espera-se que com o tempo e melhoria nos manejos estudados possam ocorrer alterações significativas nos atributos químicos e físicos do solo.

A conversão de ecossistemas naturais em áreas cultivadas promove alteração nas taxas de adição e perdas efetivas de MOS. O declínio ou acréscimo da MOS dependerá, para o mesmo ambiente edáfico, do tipo de manejo adotado após a mudança do ecossistema (BAYER et al., 2000). Se esse manejo for mantido por um longo período, os teores de MOS tendem a se estabilizar em novo equilíbrio (BAYER; MIELNICZUK, 1999). Para Stevenson (1994), os fatores de formação do solo são os determinantes primários dos processos de ciclagem de C, uma vez que exercem influência sobre o aporte de resíduos e sobre as saídas de C do mesmo.

Corroborando com os dados encontrados na Tabela 2, Viana et al. (2011) citaram que os valores de Ds em áreas de $\mathrm{MN}$ e cultivadas com pastagem, mandioca e cana de açúcar foram diferenciados em função do manejo. O menor valor de Ds foi verificado em $\mathrm{MN}$ e a maior Ds foi observada em solo cultivado com cana de açúcar, seguido daqueles cultivados com mandioca $e$ pastagem, que também não diferiram entre si. Ainda segundo Viana et al. (2011), tem sido verificado que o aumento da Ds nas áreas agrícolas está ligado ao intenso uso do solo, provavelmente, devido ao excessivo tráfego de máquinas, no caso das áreas com cana de açúcar, ou à presença de animais em condições favoráveis à compactação do solo, no caso do sistema de pastagem. Em ambos 
os casos, a perda excessiva de MOS e de COS acentuam estes efeitos.

Keller e Håkansson (2010) associaram o aumento da Ds com a redução da estabilidade do solo, o que está intrinsecamente relacionado aos menores teores de MOS. Esta informação corrobora os resultados apresentados na Tabela 2, uma vez que com a mudança do ecossistema natural da região, ou seja, a retirada da $\mathrm{MN}$ para a introdução da atividade agrícola verificou-se a diminuição na qualidade do solo. Em contrapartida, as reduções significativas no teor de MOS e no ECOT acarretaram em aumento da Ds nas áreas com manejo das culturas.

Araújo, Tormena e Silva (2004) verificaram resultados similares de Ds, MOS e ECOT, embora tenham realizado a comparação entre áreas de cultivo da cana de açúcar e de MN. Sanches et al. (1999) também evidenciaram aumento significativo da Ds em solo cultivado com laranjeiras comparado ao de MN. Em ambos os casos, os baixos valores de MOS e o intenso tráfego de máquinas foram os fatores de maior relevância para o aumento da Ds.

Os manejos das culturas agrícolas diferiram estatisticamente entre si apenas com relação ao atributo Ds (Tabela 2). Esta única diferença observada entre os sistemas avaliados demonstra a necessidade de se melhorar o manejo das próprias culturas para que as mesmas possam expressar seu máximo potencial de produção de biomassa, que consequentemente será integrada ao solo como MOS, aumentando o teor de COS e do ECOT. Além do manejo das culturas, também se deve avaliar a qualidade do manejo da fertilidade e da estrutura do solo, fundamentais para melhorar as condições de crescimento das plantas. O carbono entra no sistema por meio da atividade fotossintética, o que demonstra a importância da presença de sistemas de manejo altamente produtivos, que, associados ao adequado uso e manejo do solo, podem promover aumentos consideráveis no seu estoque de matéria orgânica (SISTI et al., 2004).

Quando a vegetação nativa é substituída por sistemas agrícolas, o ECOT é drasticamente reduzido atingindo perdas de até $50 \%$ nos primeiros $20 \mathrm{~cm}$ de profundidade do solo (ESTADOS UNIDOS, 1999). A utilização e o preparo intenso do solo, com revolvimento da camada superficial, favorecem a exposição da fração lábil da MOS à agentes oxidantes, causando sua mineralização (VIANA et al., 2011). Além disso, a dinâmica da MOS nos sistemas agrícolas também pode estar relacionada com a característica genética, diferenças varietais, estado nutricional das culturas manejadas e à forma de preparo do solo, 
além do clima e do manejo adequado dos fertilizantes, pois todos estes fatores influenciam diretamente nos processos biológicos de decomposição da MOS.

As amostras de solo coletadas em todos os sistemas de manejo das culturas estudadas apresentaram maiores valores de Ds na profundidade de $20-40 \mathrm{~cm}$ quando comparada à profundidade $0-5 \mathrm{~cm}$ (Tabela 3). Este fato pode ser explicado pelo teor significativamente maior de MOS apresentado nas profundidades de 0-5 e 5-10 $\mathrm{cm}$ do que na camada de $20-40 \mathrm{~cm}$. Costa et al. (2009) observaram correlação semelhante em áreas de pastagem e mata nativa.

Tabela 3. Densidade aparente (Ds) e matéria orgânica do solo (MOS) em quatro profundidades amostradas.

\begin{tabular}{ccc}
\hline $\begin{array}{c}\text { Profundidade } \\
(\mathrm{cm})\end{array}$ & $\begin{array}{c}\mathrm{D} \\
\left(\mathrm{g} \mathrm{cm}_{\mathrm{s}}\right)\end{array}$ & $\begin{array}{c}\text { MOS } \\
\left(\mathrm{g} \mathrm{dm}^{-3}\right)\end{array}$ \\
\hline $0-5$ & $1,27 \mathrm{~b}$ & $23,43 \mathrm{a}$ \\
$5-10$ & $1,30 \mathrm{ab}$ & $17,90 \mathrm{~b}$ \\
$10-20$ & $1,29 \mathrm{ab}$ & $15,43 \mathrm{bc}$ \\
$20-40$ & $1,43 \mathrm{a}$ & $13,10 \mathrm{c}$ \\
\hline CV(\%) & 6,68 & 22,38 \\
DMS & 0,12 & 2,74 \\
\hline
\end{tabular}

Letras iguais, minúsculas na coluna não diferem entre si a $5 \%$ de probabilidade pelo teste Tukey.

Os manejos de SSD e PST/PSTI proporcionaram maior valor de Ds quando comparados à área de $\mathrm{MN}$ e não diferiram entre si com relação aos valores de MOS, embora tenham apresentado menores valores deste parâmetro com relação à área de MN (Tabela 2). Entretanto, ao se analisar apenas a profundidade do solo, considerando todo o sistema de manejo vegetal, verifica-se que as camadas superiores $(0-5$ e 5-10 cm), por estarem mais suscetíveis às ações de tráfego frequente de máquinas e pisoteio animal, apresentaram maior teor de MOS, o que, possivelmente colaborou para minimizar seus efeitos na Ds (Tabela 3).

O maior valor de ECOT foi observado na última profundidade de solo analisada, considerando cada sistema de manejo individualmente (Tabela 4). 
Tabela 4. Estoque de carbono orgânico total (ECOT) considerando a interação entre os sistemas de manejo e as quatro profundidades de solo avaliadas.

\begin{tabular}{ccccc}
\hline \multirow{2}{*}{ Sistema Vegetal } & \multicolumn{4}{c}{ Profundidade $(\mathrm{cm})$} \\
\cline { 2 - 5 } & $0-5$ & $5-10$ & $10-20$ & $20-40$ \\
\hline SSD & $6,35 \mathrm{bB}$ & $5,83 \mathrm{aB}$ & $11,00 \mathrm{aB}$ & $22,71 \mathrm{bA}$ \\
$\mathrm{EUC}$ & $5,86 \mathrm{aB}$ & $5,01 \mathrm{aB}$ & $8,75 \mathrm{bAB}$ & $15,98 \mathrm{bA}$ \\
PST & $6,58 \mathrm{aAB}$ & $5,03 \mathrm{aB}$ & $8,48 \mathrm{bAB}$ & $14,03 \mathrm{bA}$ \\
PSTI & $7,73 \mathrm{aAB}$ & $5,26 \mathrm{aB}$ & $8,91 \mathrm{bAB}$ & $15,17 \mathrm{bA}$ \\
MN & $11,86 \mathrm{aC}$ & $12,82 \mathrm{aC}$ & $23,10 \mathrm{aB}$ & $37,54 \mathrm{aA}$ \\
\hline CV(\%) para Sistema de Manejo $=37,79 ; \mathrm{CV}(\%)$ & para & Profundidade $=32,21 ;$ DMS para colunas = 12,58 (letras \\
minúsculas); DMS para linhas = 8,50 (letras maiúsculas). Letras iguais, minúsculas na coluna e maiúsculas na linha, não
\end{tabular}

O ECOT foi superior na maior profundidade de amostragem devido ao aprofundamento do sistema radicular das plantas, proporcionando maior aporte de material orgânico e, consequentemente, maior acúmulo de COS. Segundo Gatto et al. (2011) existe aumento na produção de biomassa de raízes em profundidade em locais onde o déficit nutricional e/ou hídrico são mais acentuados. Nestas situações, as plantas tendem a alocar mais fotoassimilados para as raízes, em detrimento dos demais órgãos vegetais (GONÇALVES; MELLO, 2000), possibilitando a busca de nutrientes e água em profundidade.

Em ambientes com manejo de pastagens, grande parte do sistema radicular das gramíneas concentra-se aproximadamente na faixa de $40 \mathrm{~cm}$ de profundidade, o que garante maior aporte de matéria orgânica nas camadas superficiais (COSTA et al., 2000). Costa et al. (2009) também observaram aumento no ECOT, em profundidade, nas áreas de mata nativa e de pastagem, devido à diferença na espessura das camadas de solo avaliadas. De acordo com os autores, o aumento da Ds foi observado tanto em profundidade quanto na mudança do uso da terra, ou seja, áreas desmatadas com posterior introdução de pastagem.

Tarré et al. (2001) também mostraram que, pelo maior aporte de MOS proporcionado pelas raízes, os solos sob pastagens apresentaram teores iguais ou superiores aos encontrados em ambientes de mata, enquanto resultados obtidos por Noordwijk et al. (1997) indicaram valores de MOS mais altos nos solos provenientes de áreas de mata, proporcionados pelo maior aporte global de resíduos orgânicos vegetais. Nestes casos, o tempo e a qualidade do manejo das áreas cultivadas influenciaram na 
resposta das plantas e nos atributos químicos do solo.

Ainda com relação ao ECOT, houve aumento significativo nas amostras de solo representativas da camada de $20-40 \mathrm{~cm}$ em relação às amostras das profundidades de 0 5 e 5-10 cm, entre os sistemas vegetais avaliados, exceto para o sistema SSD na camada 0-5 (Tabela 4). O sistema de MN apresentou ECOT significativamente superior nas profundidades de $10-20 \mathrm{~cm}$ e $20-40 \mathrm{~cm}$ quando comparadas às demais profundidades. Já em relação aos sistemas de manejo com pastagens (PSTI e PST), não foi verificada diferença significativa no ECOT, demonstrando que o manejo das forrageiras nestas áreas é recente e que ainda há acúmulo de carbono no solo derivado da área de $\mathrm{MN}$ anteriormente existente no local.

A área manejada em SSD apresentou diferença significativa superior no ECOT em relação às demais áreas agrícolas apenas na profundidade de $10-20 \mathrm{~cm}$, e todas as demais formas de manejo apresentaram valores de ECOT estatisticamente menores aos encontrados na área de $\mathrm{MN}$, na profundidade de $20-40 \mathrm{~cm}$ (Tabela 4). O ECOT no SSD foi menor do que o encontrado por Lovato et al. (2004) e Gatto et al. (2011), possivelmente em função da perda superficial de carbono $(0-10 \mathrm{~cm})$ gerada pelo manejo convencional do solo no período que antecedeu a implantação do SSD. Além disso, o ECOT obtido de $10-20$ e $20-40 \mathrm{~cm}$ pode ter sido oriundo da reserva de matéria orgânica depositada pelo sistema de $\mathrm{MN}$ que formava a vegetação da área antes de sua transformação em área agrícola.

De acordo com Lovato et al. (2004), o cultivo do solo, originalmente, sob campo nativo, resultou na diminuição dos ECOT na camada de $0-17,5 \mathrm{~cm}$ de 44,76 para $32,55 \mathrm{Mg}$ $\mathrm{ha}^{-1}$ em razão do intenso revolvimento do solo com enxada rotativa e baixa adição de resíduos vegetais. Posteriormente, os autores verificaram que os ECOT na camada representativa da camada de $17,5 \mathrm{~cm}$ de profundidade foram influenciados significativamente pelo preparo do solo e sistemas de culturas e, na camada de 17,5-30 $\mathrm{cm}$ o ECOT foi pouco alterado pelo cultivo do solo. Six, Elliot e Paustian (1999) salientaram que o processo de proteção física da matéria orgânica é mais intenso em solos não revolvidos, pois a interação da mesma com os minerais formam agregados estáveis que diminuem a ação dos microrganismos decompositores, contribuindo para o acúmulo de compostos orgânicos no solo.

\section{CONCLUSÕES}

A retirada da vegetação nativa proporcionou aumento da densidade aparente e reduções no teor de matéria 
orgânica e no estoque de carbono total do solo.

Os diferentes sistemas de manejo de culturas agrícolas não melhoraram os valores dos atributos densidade aparente e matéria orgânica do solo, mas o manejo de semeadura direta na palha apresentou maior estoque de carbono total do solo na profundidade de $20-40 \mathrm{~cm}$.

\section{REFERÊNCIAS}

ADDISCOTT, T.M. Entropy and sustainability. European Journal Soil Science, v.46, n.2, p.161-168, 1995. http://dx.doi.org/10.1111/j.13652389.1995.tb01823.x

ARAÚJO, M.A.; TORMENA, C.A.; SILVA, A.P. Propriedades físicas de um latossolo vermelho cultivado e sob mata nativa. Revista Brasileira de Ciência do Solo, v.28, n.2, p.337-345, 2004. http://dx.doi.org/10.1590/s010006832004000200012

BAYER, C.; MIELNICZUK, J. Dinâmica e função da matéria orgânica. In: SANTOS, G.A.; CAMARGO, F.A.O. Fundamentos da matéria orgânica: Ecossistemas tropicais e subtropicais. Porto Alegre: Gênesis, 1999. p.9-23.

BAYER, C.; MIELNICZUK, J.; AMADO, T.J.C.; MARTIN-NETO, L. Efeito de sistemas de preparo e de cultura na dinâmica da matéria orgânica e na mitigação das emissões de $\mathrm{CO}_{2}$. Revista Brasileira de Ciência do Solo, v.24, n.3, p.599-607, 2000.

BLANCO-CANQUI, H.; STONE, L.R.; SCHLEGEL, A.J.; LYON, D.J.; VIGIL, M.F.; MIKHA, M.M.; STAHLMAN, P.W.; RICE, C.W. No-till induced increase in organic carbon reduces maximum bulk density of soils. Soil Science Society American Journal, v.73, n.6, p.1871-1879, 2009.

http://dx.doi.org/10.2136/sssaj2008.0353

CARDOSO, A.; POTTER, R.; DEDECEK, R.A. Estudo comparativo da degradação de solos pelo uso agrícola no Noroeste do estado do Paraná. Pesquisa Agropecuária Brasileira, v.27, n.3, p.349-353, 1992.

CARVALHO, J.L.N.; AVANZI, J.C.; SILVA, M.L.N.; MELLO, C.R.; CERRI, C.E.P. Potencial de sequestro de carbono em diferentes biomas do Brasil. Revista Brasileira de Ciência do Solo, v.34, n.2, p.277-289, 2010. http://dx.doi.org/10.1590/S010006832010000200001.

CONCEIÇÃO, P.C.; BOENI, M.; DIECKOW, J.; BAYER, C.; MIELNICZUK, J. Fracionamento densimétrico com politungstato de sódio no estudo da proteção física da matéria orgânica em solos. Revista Brasileira de Ciência do Solo, v.32, n.2, p.541-549, 2008. http://dx.doi.org/10.1590/S010006832008000200009.

COSTA, O.V.; COSTA, L.M.; FONTES, L.E.F.; ARAÚJO, Q.R.; KER, J.C.; NACIF, P.G.S.; Cobertura do solo e degradação de pastagens em área de domínio de chernossolos no sul da Bahia. Revista Brasileira de Ciência do Solo, v.24, n.4, p.843-856, 2000.

COSTA, O.V.; CANTARUTTI, R.B.; FONTES, L.E.F.; COSTA, L.M; NACIF, P.G.S; FARIA, J.C. Estoque de carbono do solo sob pastagem em área de tabuleiro costeiro no sul da Bahia. Revista Brasileira de Ciência do Solo, v.33, n.5, p.1137-1145, 2009. http://dx.doi.org/10.1590/s010006832009000500007

ELBERT, B.H.; BETTANY, J.R. Calculation of organic matter and nutrients stored in soils under contrasting management regimes. Canadian Journal Soil Science, v.75, n.4, 
p.529-538, 1995.

http://dx.doi.org/abs/10.4141/cjss95-075

EMBRAPA. Manual de métodos de análise de solo. Rio de Janeiro: Centro Nacional de Pesquisa de Solos, 1997. 212 p.

EMBRAPA. Sistema brasileiro de classificação de solos. Rio de Janeiro: Centro Nacional de Pesquisa de Solos, 2006. 306 p.

ESTADOS UNIDOS. Department of energy, sequestration of carbon: State of the Science. Washington, 1999.

FEDDEMA, J.J. Estimated impacts of soil degradation on the african water balance and climate. Climate Research, v.10, n.2, p.127141, 1998. http://doi:10.3354/cr010127

GATTO, A.; BARROS, N.F.; NOVAIS, R.F.; SILVA, I.R.; LEITE, H.G.; VILLANI, E.M. A. Estoque de carbono na biomassa de plantações de Eucalipto na região centroleste do estado de Minas Gerais. Revista Árvore, v.35, n.4, p.895-905, 2011. http://dx.doi.org/10.1590/S0100-

67622011000500015

GONÇALVES, J.L.M.; MELLO, S.L.M.O. Sistema radicular das árvores. In: GONÇALVES, J.L.M.; BENEDETTI, V.E. Nutrição e fertilização florestal. Piracicaba: IPEF, 2000. p.221-267.

KELLER, T.; HAKÅNSSON, I. Estimation of reference bulk density from soil particle size distribution and soil organic matter content. Geoderma, v.154, n.3-4, p.398-406, 2010. http://dx.doi.org/10.1016/0167

LOVATO, T.; MIELNICZUK, J.; BAYER, C.; VEZZANI, F. Adição de carbono e nitrogênio e sua relação com os estoques no solo e com o rendimento do milho em sistemas de manejo. Revista Brasileira de Ciência do Solo, v.28, n.1, p.175-187, 2004. http://dx.doi.org/10.1590/S0100-

06832004000100017
NOORDWIJK, M.V.; CERRI, C.C.; WOOMER, P.L.; NUCROHO, K.; BERNOUX, M. Soil carbon dynamics in the humid tropical forest zone. Geoderma, v.79, n.1-4, p.187-225, 1997. http://dx.doi.org/10.1016/S00167061(97)00042-6

OLIVEIRA, J.B. Solos do Estado de São Paulo: descrição das classes registradas no mapa pedológico. Campinas: Instituto Agronômico, 1999. 112p.

PAGLIAI, M.; VIGNOZZI, N.; PELLEGRINI, S. Soil structure and the effect of management practices. Soil Tillage Research, v.79, n.1, p.131-143, 2004. http://dx.doi.org/10.1016/j.still.2004.07.002

RAIJ, B.V.; ANDRADE, J.C.; CANTARELLA, H.; QUAGGIO, J.A. Análise química para avaliação da fertilidade de solos tropicais. 1. ed. Campinas: Instituto Agronômico, 2001. $285 p$.

SANCHES, A.C.; SILVA, A.P.; TORMENA, C.A.; RIGOLIN, A.T. Impacto do cultivo de citros em propriedades químicas, densidade do solo e atividade microbiana de um podzólico vermelho-amarelo. Revista Brasileira de Ciência do Solo, v.23, n.1 p.91-99, 1999.

SISTI, C.P.J. SANTOS. H.P.; KOHHANN, R.; ALVES, B.J.R.; URQUIAGA, S.; BODDEY, R.M. Change in carbon and nitrogen stocks in soil under 13 years of conventional or zero tillage in southern Brazil. Soil and Tillage Research, v.76, n.1, p.39-58, 2004. http://dx.doi.org/10.1016/j.still.2003.08.007

STEVENSON, F.J. Humus chemistry: genesis, composition, reactions. 2. ed. New York: John Wiley \& Sons, 1994. 496p.

SIX, J.; ELLIOTT, E.T.; PAUSTIAN, K. Aggregate and soil organic matter dynamics under conventional and no-tillage systems. Soil Science Society American Journal, v.63, n.5, 
p.1350-1358,

1999.

http://dx.doi.org/10.2136/sssaj1999.635135

$0 \mathrm{x}$

STONE, L.F.; SILVEIRA, P.M. Efeitos do sistema de preparo e da rotação de culturas na porosidade e densidade do solo. Revista

Brasileira de Ciência do Solo, v.25, n.2, p.395-401, 2001.

TARRÉ, R.; MACEDO, R.; CANTARUTTI, R.B.; REZENDE, C.P.; PEREIRA, J.M.; FERREIRA, E.; ALVES, B.J.R.; URQUIAGA, S.; BODDEY, R.M. The effect of the presence of a forage legume on nitrogen and carbon levels in soils under Brachiaria pastures in the Atlantic forest region of the South of Bahia, Brazil. Plant Soil, v.234, n.1, p.15-16, 2001. http://dx.doi.org/10.1023/A:1010533721740

TISDALL, J.M.; OADES, J.M. Organic matter and water-stable aggregates in soils. Journal of Soil Science, v.33, n.2, p.141-163, 1982. http://dx.doi.org/10.1111/j.1365-

2389.1982.tb01755.x

VIANA, E.T.; BATISTA, M.A.; TORMENA, C.A.; COSTA, A.C.S.; INOUE, T.T. Atributos físicos e carbono orgânico em latossolo vermelho sob diferentes sistemas de uso e manejo. Revista Brasileira de Ciência do Solo, v.35, n.6, p.2015-2114, 2011. http://dx.doi.org/10.1590/S010006832011000600025

WALKLEY, A. BLACK, I.A. An examination of the Degtjareff method for determining soil organic matter, and proposed modification of the chromic acid titration method. Soil Science, v.37, n.1, p.29-38, 1934.

ZINN, Y.L.; LAL, R.; RESCK, D.V.S. Texture and organic carbon relations described by a profile pedotransfer function for Brazilian Cerrado soils. Geoderma, v.127, n.1-2, p.68173, 2005. http://dx.doi.org/10.1016/j.geoderma.2005.0 2.010
Recebido para publicação em 18/02/2013

Revisado em 17/02/2014

Aceito em 15/05/2014 